\title{
光催化氧化还原体系中硝酮与芳香叔胺的自由基偶联反应
}

\author{
刘玉成郑啸* 黄培强* \\ (厦门大学化学化工学院化学系 福建省化学生物学重点实验室 厦门 361005)
}

\begin{abstract}
摘要 通过氮 $\alpha$-位碳自由基构造氮 $\alpha$-位碳一碳键是合成含氮有机化合物的重要方法. 近期, 利用可见光催化氧化芳香叔 胺一氮 $\alpha$-位去质子化形成氮 $\alpha$-位碳自由基的原理发展了一系列新颖的自由基加成(偶联)反应，成为氮 $\alpha$-位碳自由基化 学发展的重要方向. 本文应用 Ir-催化剂, 实现了光催化氧化还原体系中硝酮与芳香叔胺的自由基偶联反应, 高效地合 成 $\beta$-氨基羟胺化合物. 该反应条件温和、操作简单，具有较高的原子经济性，且对于各种链状、环状以及手性硝酮都具 有良好的适用性, 产物可方便地转化为重要的邻二胺化合物.
\end{abstract}

关键词 氮 $\alpha$-位碳自由基; 光催化氧化还原; 偶联反应; 芳香叔胺; 硝酮

\section{Photoredox Catalysis for the Coupling Reaction of Nitrones with Aromatic Tertiary Amines}

\author{
Liu, Yu-Cheng Zheng, Xiao* Huang, Pei-Qiang* \\ (Department of Chemistry, Fujian Provincial Key Laboratory of Chemical Biology, College of Chemistry and Chemical \\ Engineering, Xiamen University, Xiamen, Fujian 361005, China)
}

\begin{abstract}
Carbon-carbon bond formation at $\alpha$-amino carbon based on $\alpha$-aminoalkyl radicals is an essential transformation in the synthesis of nitrogen-containing compounds. Recently, some novel photoredox catalytic protocols for this goal have been developed, in which $\alpha$-aminoalkyl radicals were generated from a sequential oxidation/ $\alpha$-deprotonation of aromatic tertiary amines. Inspired by these studies and based on our previous works, we have developed the cross-coupling reaction of nitrones with aromatic tertiary amines via visible light photoredox catalysis. This method features a radical addition of $\alpha$-aminoalkyl radicals to nitrones with advantages of simple operation, mild conditions, atom economy, a broad scope of nitrone substrates; and allows for an easy access to $\beta$-amino hydroxylamines, which could be readily converted into vicinal diamines. Compared with the UV-excited organophotosensitizer-promoted coupling reaction of nitrones with tertiary amines, visible light is a more safe and convenient light source, the photo-excited electron transfer (PET) by $1 \mathrm{~mol} \%$ of Ir-photocatalyst is more efficient. In addition, nitrones exclusively server as excellent radical acceptors thus with a broader range of structures. A general procedure of this coupling reaction is as follows: To a $25 \mathrm{~mL}$ Schlenk tube equipped with a magnetic stir bar were added a nitrone $(0.30 \mathrm{mmol})$, a tertiary amine $(0.90 \mathrm{mmol}), \operatorname{Ir}(\mathrm{ppy})_{2}(\mathrm{dtbbpy}) \mathrm{PF}_{6}(0.003 \mathrm{mmol}, 1.0 \mathrm{~mol} \%)$ and $\mathrm{K}_{2} \mathrm{HPO}_{4}(0.06 \mathrm{mmol}$, $20 \mathrm{~mol} \%)$. After being evacuated and backfilled with argon for three times, DMSO $(3 \mathrm{~mL})$ was added to the tube. Then the tube was placed approximately $7 \mathrm{~cm}$ away from a $12 \mathrm{~W}$ blue LEDs, and the reaction mixture was stirred at r.t. under an argon atmosphere for $24 \mathrm{~h}$. The reaction was quenched with saturated aqueous $\mathrm{NaHCO}_{3}(25 \mathrm{~mL})$, and the mixture was extracted with dichloromethane $(\mathrm{DCM}, 20 \mathrm{~mL} \times 3)$. The combined organic layers were washed with brine, dried over anhydrous $\mathrm{Na}_{2} \mathrm{SO}_{4}$, filtered and concentrated under reduced pressure. The residue was purified by flash column chromatography on silica gel to afford the desired cross-coupling product $\beta$-amino hydroxylamine.
\end{abstract}

Keywords $\alpha$-aminoalkyl radicals; photoredox catalysis; coupling reaction; aromatic tertiary amine; nitrone

\section{1 引言}

在有机合成中, 氮 $\alpha$-位碳自由基是一类重要的有机 活性中间体，被广泛用于构建氮 $\alpha$-位碳-碳键，并被应 用于含氮天然产物和生物活性分子的合成 ${ }^{[1]}$. 近年来, 可见光催化的自由基反应已成为有机合成化学发展的
前沿热点 ${ }^{[2]}$. MacMillan 小组 ${ }^{[3]}$ 率先利用可见光催化氧化 一氮 $\alpha$-位去质子化的原理将芳香叔胺转化为氮 $\alpha$-位碳 自由基，进而实现了叔胺氮 $\alpha$-位的(杂)芳基化. 在此之 后, 利用这一原理获得的氮 $\alpha$-位碳自由基也被拓展用于 与 $\alpha, \beta$-不饱和化合物 ${ }^{[4]}$ 、偶氮二碳酸二叔丁酯 ${ }^{[5]}$ 、亚胺 化合物 ${ }^{[6]}$ 、羰基化合物 ${ }^{[66,7]}$ 和高价碘氰基试剂 ${ }^{[8]}$ 等进行自

*E-mail: zxiao@xmu.edu.cn, pqhuang@xmu.edu.cn

Supporting information for this article is available free of charge via the Internet at http://sioc-journal.cn.

Project supported by the National Key Research and Development Program of China (No. 2017YFA0207302), the National Natural Science Foundation of China (Nos. 21672175, 91856110, 21332007, 21472153), and the Program for Changjiang Scholars and Innovative Research Team in University (PCSIRT) of Ministry of Education, China.

项目受国家重点研发计划(No. 2017YFA0207302)、国家自然科学基金(Nos. 21672175, 91856110, 21332007, 21472153)和教育部长江学者和创新团队发 展计划资助。 
由基加成(偶联)反应, 发展了一系列在氮 $\alpha$-位形成碳碳键的新方法(图式 1a) ${ }^{[1 \mathrm{~d}]}$. 另一方面, Itoh 等 ${ }^{[9]}$ 于 2015 年报道了紫外光激发下, 由叔胺产生的氮 $\alpha$-位碳自由基 与硝酮的加成反应(图式 $1 b$ ). 值得指出的是, 可见光催 化的方法大多是在早期紫外光激发、光敏剂促进的方 法 $^{[1 \mathrm{c}, 10]}$ 基础上发展起来的; 在使用了更为安全、方便的 光源和更为高效的光催化剂后, 在氮 $\alpha$-位碳自由基的产 生机制和偶联方式上也有新的拓展. 基于这样的研究思 路, 我们关注到该反应中, 紫外光激发的二苯甲酮类光 敏剂和硝酮共同促进了叔胺氮 $\alpha$-位碳自由基中间体的 生成. 近十年来, 本课题组也通过二碘化钐参与和一氯 二茂钛催化, 发展了一系列基于氮 $\alpha$-位碳自由基的反应 方法 ${ }^{[11]}$, 并于近期发展了光催化与路易斯酸协同催化下
硝酮与芳香醛的不对称还原偶联反应，对映选择性地合 成了邻氨基醇化合物(图式 $1 \mathrm{c})^{[12]}$. 在此基础上, 我们设 想如果将芳香叔胺经光催化氧化一去质子化生成的氮 $\alpha$-位碳自由基(I) 以硝酮捕获, 就有可能建立一个无需额 外共氧化剂或共还原剂的光催化氧化还原体系, 高效地 合成 $\beta$-氨基差胺化合物，这类化合物又可方便地转化为 重要的邻二胺化合物(图式 1d) ${ }^{[13,14]}$. 在此我们报道该研 究的初步结果.

\section{2 结果与讨论}

\section{1 硝酮与芳香叔胺的自由基偶联反应条件篮选}

首先, 参考 Rueping 等 ${ }^{[6 b]}$ 的报道, 结合实验室前期

a) Photocatalysis for radical reactions of aromatic tertiary amines

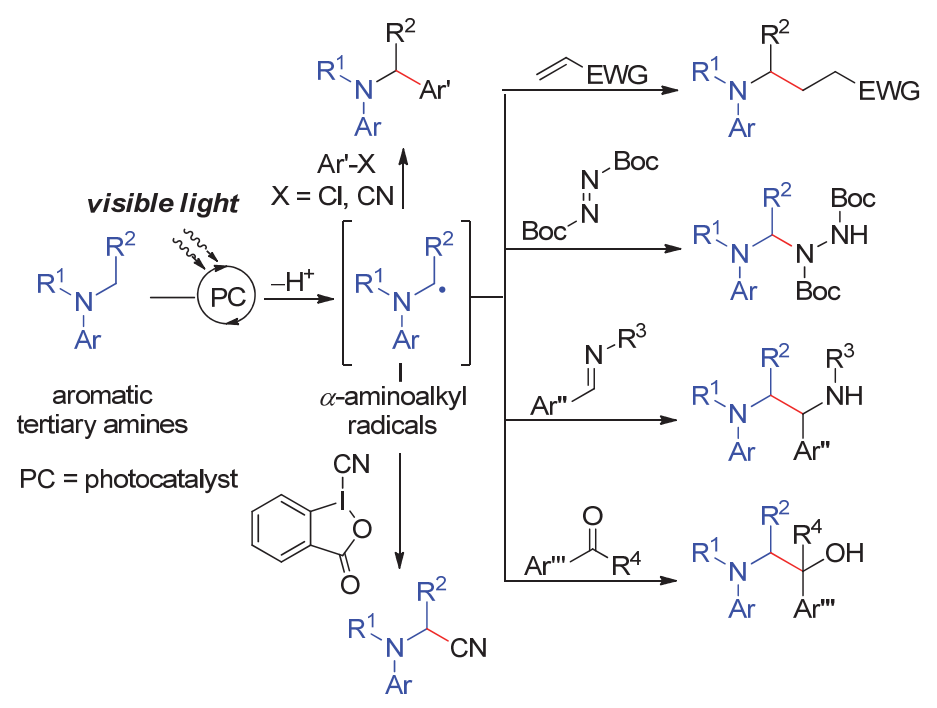

b) UV-excited radical coupling of nitrones with tertiary amines

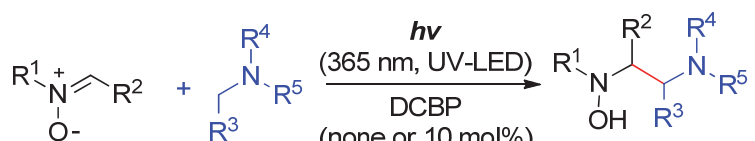

$$
\begin{aligned}
& \text { nitrones tertiary amines } 10 \mathrm{~mol} \%) \text {-amino hydroxylamines } \\
& \text { DCBP }=4,4 \text { '-dichlorobenzophenone }
\end{aligned}
$$

c) Previous work in our lab

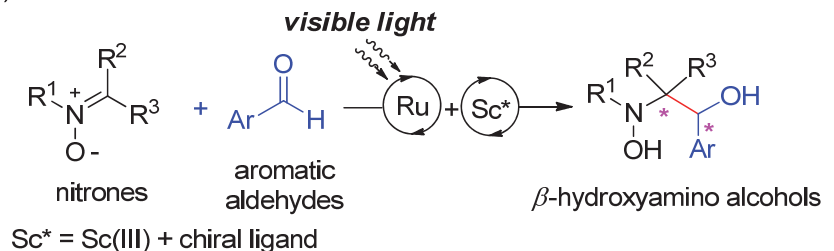

d) This work

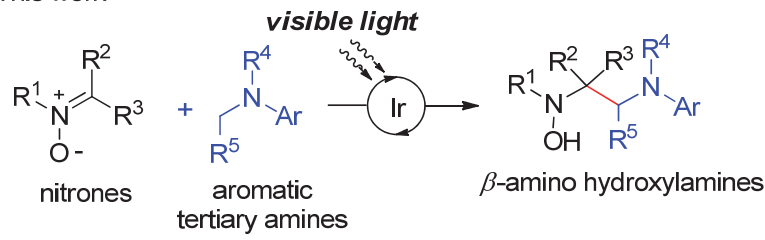

图式 1 可见光催化氧化还原体系中硝酮与芳香叔胺的自由基偶联反应设计

Scheme 1 The design of cross-coupling of nitrones with aromatic tertiary amines via visible light photoredox catalysis 
的研究 ${ }^{[12]}$, 我们以 $\mathrm{Ru}(\mathrm{bpy})_{3}\left(\mathrm{PF}_{6}\right)_{2}$ 为光催化剂, 碳酸铯 $\left(\mathrm{Cs}_{2} \mathrm{CO}_{3}\right)$ 为碱, 二甲亚砜(DMSO)为溶剂, 在 $12 \mathrm{~W}$ LEDs 蓝光照射下, 考察了硝酮 $\mathbf{1 a}$ 和商品化的 $N, N$-二甲基苯 胺 2a 的模型反应，以 $14 \%$ 的产率获得了交叉偶联产物， $\beta$-氨基着胺 3aa(表 1, Entry 1). 之后, 经过对反应条件的 探索和优化(详见 SI, 表 S1), 我们确定了反应的最佳条 件为: 硝酤(1.0 equiv.)、芳香叔胺(3.0 equiv.)、光催化剂 为 $\left[\operatorname{Ir}(\mathrm{ppy})_{2} \mathrm{dtbbpy}\right] \mathrm{PF}_{6}(1.0 \mathrm{~mol} \%)$ 、碱为 $\mathrm{K}_{2} \mathrm{HPO}_{4}(20$ $\mathrm{mol} \%)$ 、溶剂为 $\operatorname{DMSO}\left(c_{\text {nitrone }}=0.1 \mathrm{~mol} / \mathrm{L}\right)$ 、光源为 $12 \mathrm{~W}$ LEDs 蓝光, 反应时间 $24 \mathrm{~h}$. 在此条件下, $\beta$-氨基羟胺产 率可达 84\%(表 1, Entry 2). 进而, 通过对照实验证明光 照、光催化剂和碱都是反应得以进行不可或缺的条件 (表 1, Entries 3 5).

\section{2 硝酮底物普适性的研究}

在确定优化反应条件后, 我们首先对不同的硝酮底 物进行考察(表 2). 结果显示对于链状硝酮, 无论碳端 $\left(\mathrm{R}^{2} / \mathrm{R}^{3}\right)$ 和氮端 $\left(\mathrm{R}^{1}\right)$ 是烷基或者芳基取代, 都可顺利与 $N, N$-二甲基苯胺 $2 \mathbf{a}$ 反应, 以中等到较高的产率得到交 叉偶联的产物. 醛缩硝酮 $\left(R^{2}=H\right)$ 碳端 $\left(R^{3}\right)$ 或是氮端 $\left(R^{1}\right)$ 的取代基位阻增大，反应产率降低(3aa vs. $3 \mathbf{b} \sim 3 \mathbf{f}$ and 3h vs. 3i and 3j); 碳端的立体效应大于氮端的立体效应 (3i and 3j vs. 3k); 碳端双取代, 即酮缩硝酮(1g)的位阻 效应大于醛缩硝酮 $(1 \mathrm{a} \sim 1 \mathrm{c}, 1 \mathrm{e}, 1 \mathrm{f})$. 此外, 我们观察到 环状硝酮具有较高的反应性，都能以较高的产率得到预 期的偶联产物 $(3 \mathrm{I} \sim 3 \mathbf{p})$; 由 $L$-苹果酸和 $D$-酒石酸衍生

表 1 对照实验

Table 1 Control experiments

$$
\text { (1.0 equiv.) }
$$

\begin{tabular}{ccc}
\hline Entry & Variation from standard condition & Yield/\% \\
\hline 1 & $\mathrm{Ru}(\mathrm{bpy})_{3}\left(\mathrm{PF}_{6}\right)_{2} / \mathrm{Cs}_{2} \mathrm{CO}_{3}$ & 14 \\
2 & & 84 \\
3 & No Photocatalyst & 0 \\
4 & No LEDs & 0 \\
5 & $\mathrm{No} \mathrm{K}_{2} \mathrm{HPO}_{4}$ & 0 \\
\hline
\end{tabular}

表 2 硝酮与 $N, N$-二甲基苯胺 $\mathbf{2 a}$ 的交叉偶联反应

Table 2 Cross-coupling of nitrones with $N, N$-dimethylaniline 2a

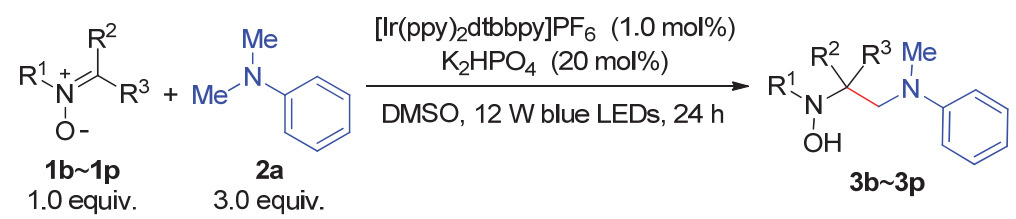<smiles>CC(C)=CCCC(C)CC(CN(Cc1ccccc1)c1ccccc1)N(O)c1ccccc1N(Cc1ccccc1)C(c1ccccc1)N(O)c1ccccc1</smiles><smiles>CC(CN(C)c1ccccc1)N(C)O</smiles><smiles>CCCC(CN(C)c1ccccc1)N(C)O</smiles><smiles>CN(O)C(CN(c1ccccc1)c1ccccc1)c1ccccc1</smiles><smiles>CN(CCN(O)C(C)(C)C)c1ccccc1</smiles><smiles>C[13C](=O)CC1CCCN1O</smiles><smiles>CC1(C)CCC(CN([O-])c2ccccc2)N1O</smiles><smiles>CC1CCN(O)C(CN(C)c2ccccc2)C1</smiles><smiles>C#Cc1ccc(N(CC(=O)N(Cc2ccccc2)c2ccccc2)CC2C(O)CCN2O)cc1</smiles>
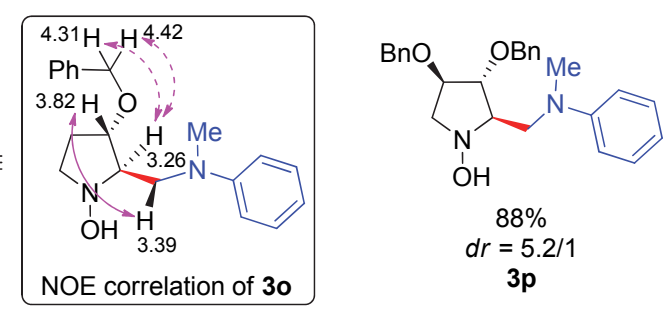
得到的手性环状硝酮 $(10,1 p)$ 与 $2 \mathrm{a}$ 的反应也得到了较高 的产率和适中的非对映立体选择性 $(\mathbf{3 o}, \mathbf{3 p})$. NOE 实验 显示硝酤 10 与叔胺 $\mathbf{2 a}$ 反应得到的是 2,3 -反式为主的产 物 30 .

\section{3 芳香叔胺普适性的研究}

接着, 我们对芳香叔胺进行了拓展. 从表 3 中可以 发现叔胺芳环上无取代时, 硝酮 $1 \mathrm{a}$ 与其的偶联反应能 得到较高的产率(3aa vs. 3ab 3ai and 3al vs. 3am \& 3an). 对于苯环对位取代的芳香叔胺, 无论供电子取代 基, 还是吸电子取代基都会降低反应的产率(3aa vs. 3ab, 3ac and 3aa vs. 3ad 3af). 我们推测产生这一现象 的原因是苯环对位带供电子基团时有利于芳香叔胺的 氧化, 也有利于芳香叔胺自由基正离子的稳定, 但却使 自由基正离子氮 $\alpha$-位 $\mathrm{C}-\mathrm{H}$ 键的酸性减弱, 不利于氮 $\alpha$ 位去质子化生成氮 $\alpha$-位碳自由基, 从而使得反应产率相 较无取代芳香叔胺明显降低; 而苯环对位带吸电子基团 时, 虽然有利于增强芳香叔胺自由基正离子氮 $\alpha$-位 $\mathrm{C}$ $\mathrm{H}$ 键的酸性, 利于氮 $\alpha$-位去质子化生成氮 $\alpha$-位碳自由
基，但是却不利于叔胺氧化，也不利于叔胺自由基正离 子的稳定，因此反应产率相较无取代芳香叔胺也降低. 另外，我们发现，取代基芳基对自由基正离子和氮 $\alpha$-位 碳自由基的稳定性越好，偶联反应的产率越高(3aj vs. 3aa), 䒺基取代可得到 90\%的偶联产率. 值得一提的是, 该反应对芳香叔胺芳环上取代的卤素和醛基都具有较 好的耐受性, $N$-苯基吡咯烷和哌啶也可以适中的产率得 到氮 $\alpha$-位胺烷基化产物(3ao, 3ap), 但 $N, N$-二甲基-4-硝 基苯胺(2q)和 $N, N$-二甲基-4-氨基吡啶(DMAP, 2r)都无 法反应得到预期的产物, 推测可能是它们的氧化电势较 高, 难以被激发态的光催化剂氧化产生自由基正离子.

\section{4 反应机理研究}

我们也对反应的机理进行了初步探讨。首先，在表 2 中，碳端为环丙基的硝酮 $1 \mathrm{c}$ 与芳香叔胺 $2 \mathbf{a}$ 的反应能 得到预计的产物 3c，而没有发现环丙基开环的产物. 当 将等化学计量的硝酮 $1 \mathrm{a}$ 和甲基乙烯基酮(4)混合与芳香 叔胺 $2 \mathrm{a}$ 反应时，可分别以 $48 \%$ 和 $40 \%$ 的产率得到相应 交叉偶联的产物 $3 \mathrm{aa}$ 和 5 (图式 2). 这些结果表明反应是

表 3 硝酮 1a 与芳香叔胺的交叉偶联反应

Table 3 Cross-coupling of nitrone 1a with aromatic tertiary amines

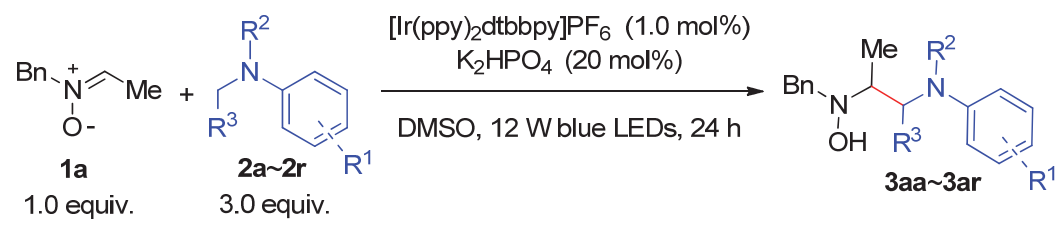

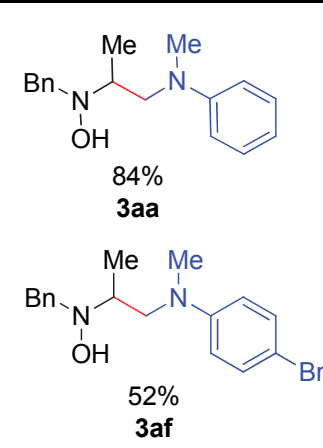<smiles>CC(CN(Cc1ccccc1)c1ccccc1)N(O)Cc1ccccc1</smiles>

3ak
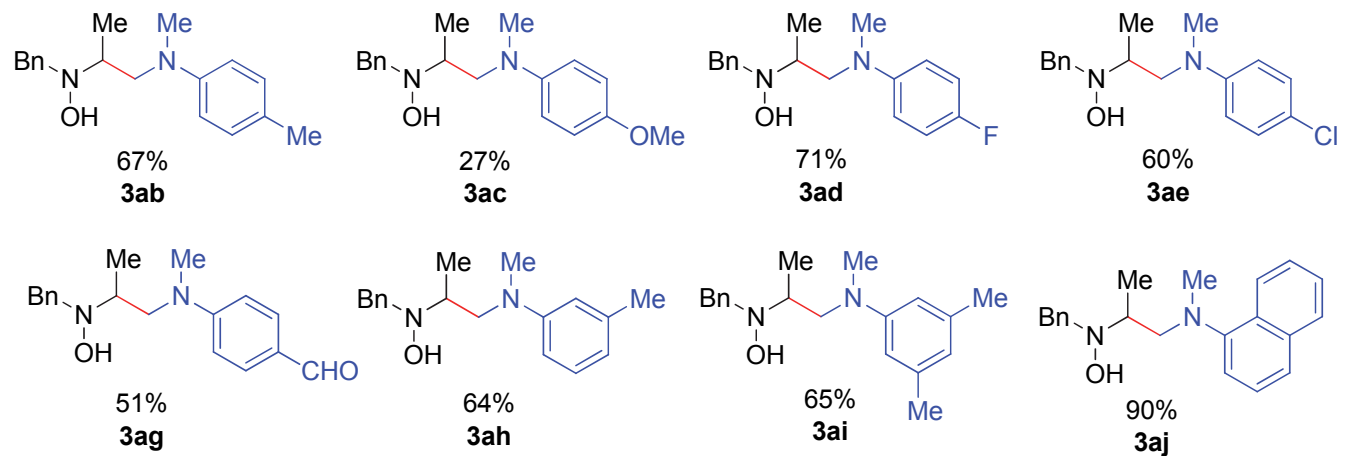<smiles>CC(CN(C)c1cccc2ccccc12)N(O)Cc1ccccc1</smiles><smiles>CC(CN(c1ccccc1)c1ccccc1)N(O)Cc1ccccc1</smiles><smiles>CC(C1CCCCN1c1ccccc1)N(O)Cc1ccccc1</smiles>

$68 \%$

$d r=1.3 / 1$

3ap
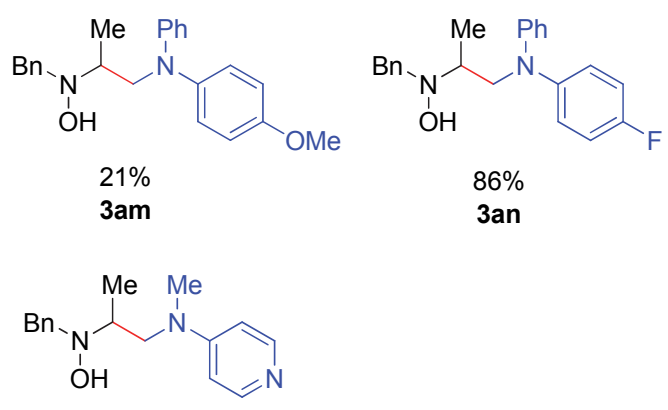

$0 \%$
$0 \%$

3aq<smiles>CC(CN(C)c1ccc([N+](=O)[O-])cc1)N(O)Cc1ccccc1</smiles>$$
\text { q }
$$ 
通过芳香叔胺产生的氮 $\alpha$-位碳自由基与硝酮加成进行 的.

其次, 我们观察到反应中芳香叔胺大量剩余, 当 0.3 $\mathrm{mmol}$ 硝酮 $\mathbf{1 a}$ 与 $0.9 \mathrm{mmol}$ 芳香叔胺 $\mathbf{2 a}$ 反应, 最终可回收 $0.57 \mathrm{mmol}$ 芳香叔胺 $\mathbf{2 a}$ (图式3). 这表明过量的芳香叔胺 在反应中并不是作为共还原剂, 反应很可能是在光催化 氧化一还原体系中进行的. 另外, 即使加大碱的用量, 叔 胺的用量也基本无法减少, 这表明过量的叔胺也许不是 作为碱, 而可能是通过提高反应体系的电子传递效率, 提高了反应的产率; 但也可能是芳香叔胺形成自由基的 效率太低, 因而需要大过量.

综上，参考文献中已报道的光催化产生芳香叔胺氮 $\alpha$-位碳自由基的机理, 推测本文反应的可能机理应如图 式4所示. 考虑到反应中只需用到催化量的碱, 推测芳 香叔胺氮 $\alpha$-位碳自由基I与硝酮加成后产生的自由基 III 可能被二价铱络合物还原为羟胺负离子 IV, 进而质子 化得到产物, 在完成光催化剂氧化还原循环的同时也完
成了碱的催化循环(图式4, route A). 当然，自由基III也 有可能直接对叔胺的自由基正离子II进行氢抽取得到产 物并生成亚胺鎓离子 $\mathbf{V}$, 然后亚胺鎓离子被二价铱络合 物还原生成叔胺氮 $\alpha$-位碳自由基II, 同时再生基态光催 化剂(图式4, route B). 值得指出的是, 在可见光催化的 偶联反应中, 铱络合物光催化剂的用量较低( $1 \mathrm{~mol} \%)$, 电子转移效率较高; 硝酮只是作为自由基受体，因此， 硝酮底物的适用范围较广. 而在紫外光激发下，硝酮不 仅是作为自由基受体，其激发态还可促进叔胺形成氮 $\alpha$ 位碳自由基. 反应在硝酮和光敏剂的共同促进下适用于 芳香叔胺和脂肪叔胺，但对硝酮结构必然有所要求，这 对硝酮底物的适用范围就有一定影响. 此外, 除了光敏 剂的电子转移效率影响外, 二苯甲酮类光敏剂在反应中 会被一些副反应所消耗，例如自身的频哪偶联和与叔胺 氮 $\alpha$-位碳自由基的交叉偶联, 因此, 光敏剂(10 mol\%)和 叔胺(5 equiv.)的用量都较高 ${ }^{[9]}$.

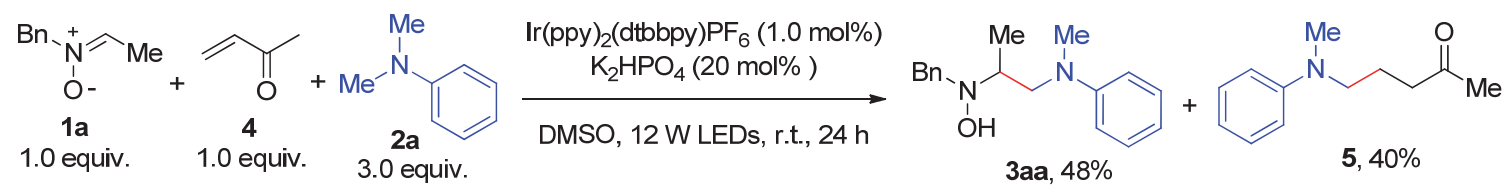

图式 2 氮 $\alpha$-位碳自由基的捕获实验

Scheme 2 The trapping experiment of $\alpha$-aminoalkyl radical

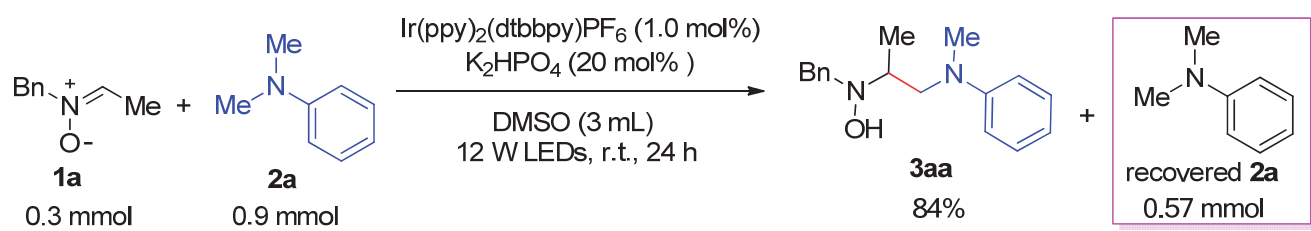

图式 3 芳香叔胺的回收实验

Scheme 3 Recovery of tertiary amine at the end of the reaction

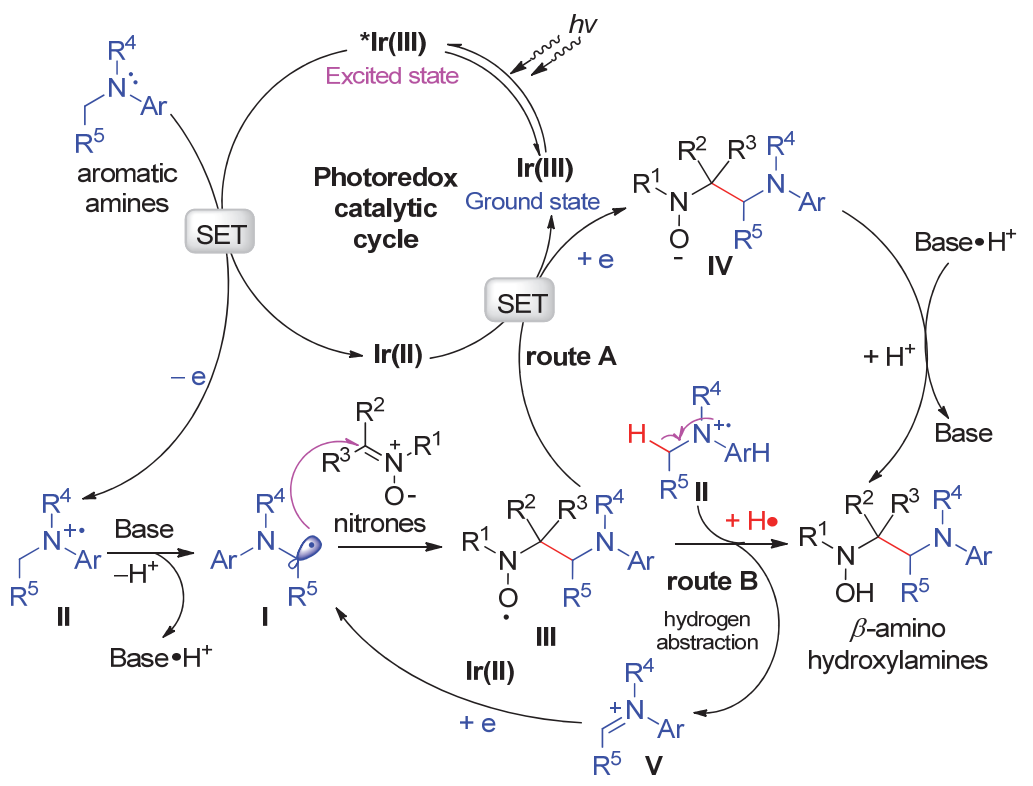

图式 4 推测的反应机理

Scheme 4 The proposed mechanism 


\section{3 结论}

总之, 我们研究了可见光催化氧化还原体系中硝酮 与芳香叔胺的交叉偶联反应，基于硝酮捕获氮 $\alpha$-位碳自 由基的机制, 发展了一种直接合成邻二胺前体化合物 $\beta$ 胺基差胺的方法. 该反应条件温和、操作简单, 具有较 高的原子经济性, 且对于各种链状、环状以及手性硝酮 都具有良好的适用性.

\section{References}

[1] (a) Renaud, P.; Giraud, L. Synthesis 1996, 913; (b) Hart, D. in Radicals in Organic Synthesis; Renaud, P.; Sibi, M. P. Eds. Weinheim, Germany: Wiley-VCH, 2001, Vol. 2: pp 279; (c) Aurrecoechea, J. M.; Suero, R. Arkivoc 2004, part xiv, 10, at www.arkat-usa.org; (d) Nakajima, K.; Miyake, Y.; Nishibayashi, Y. Acc. Chem. Res. 2016, $49,1946$.

[2] For some selected reviews on visible light photocatalysis, see: (a) Prier, C. K.; Rankic, D. A.; MacMillan, D. W. C. Chem. Rev. 2013, 113, 5322; (b) Skubi, K. L.; Blum, T. R.; Yoon, T. P. Chem. Rev. 2016, 116, 10035; (c) Fagnoni, M.; Dondi, D.; Ravelli, D.; Albini, A. Chem. Rev. 2007, 107, 2725; (d) Beatty, J. B.; Stephenson, C. R. J. Acc. Chem. Res. 2015, 48, 1474; (e) Tellis, J. C.; Kelly, C. B.; Primer, D. N.; Jouffroy, M.; Patel, N. R.; Molander, G. A. Acc. Chem. Res. 2016, 49, 1429; (f) Ghosh, L.; Marzo, L.; Das, A.; Shaikh, R.; König, B. Acc. Chem. Res. 2016, 49, 1566; (g) Chen, J.-R.; Hu, X.-Q.; Lu, L.-Q.; Xiao, W.-J. Acc. Chem. Res. 2016, 49, 1911; (h) Arora, A.; Weaver, J. D. Acc. Chem. Res. 2016, 49, 2273; (i) Li, W.; Zheng, X.-Y.; Zeng, J.-G.; Cheng, P. Chin. J. Org. Chem. 2017, 37, 1 (in Chinese); (刘薇, 郑昕宇, 曾建国, 程辟, 有机化学, Chin. J. Org. Chem. 2017, 37, 1; (j) Wang, D.-H.; Zhang, L.; Luo, S.-Z. Acta Chim. Sinica 2017, 75, 22 (in Chinese); (王德红, 张龙, 罗三中, 化学学报, 2017, 75, 22); (k) Song, H.; Liu, X.-Y.; Qin, Y. Acta Chim. Sinica 2017, 75, 1137 (in Chinese); (宋影, 刘小宇, 秦勇, 化学学报, 2017, 75, 1137); (1) Cheng, X.-K.; Hu, X.-G.; Lu, Z. Chin. J. Org. Chem. 2017, 37, 251 (in Chinese); (程骁恺, 胡新根, 陆展, 有机化学, 2017, 37, 251; (m) Wu, J.; Li, J.-W.; Li, H.; Zhu, C.-Y. Chin. J. Org. Chem. 2017, 37, 2203 (in Chinese); (吴江, 李嘉 雯，李昊，朱纯银，有机化学，2017，37，2203); (n) Liu，Y.-H.; Dong, W. Chin. J. Chem. 2017, 35, 1491; (o) Ruan, L.-H.; Dong, Z.-C.; Chen, C.-X.; Wu, S.; Sun, J. Chin. J. Org. Chem. 2017, 37 , 2544 (in Chinese); (阮利衡, 董振诚, 陈春欣, 吴爽, 孙京, 有机 化学, 2017, 37, 2544); (p) Yi, H.; Zhang, G.-T.; Wang, H.-M.; Huang, Z.-Y.; Wang, J.; Singh, A. K.; Lei, A.-W. Chem. Rev. 2017,
117, 9016; (q) Ye, H.; Xiao, C.; Lu, L.-Q. Chin. J. Org. Chem. 2018 38, 1897 (in Chinese); (叶辉, 肖聪, 陆良秋, 有机化学, 2018, 38, 1897); (r) Xu, W.-X.; Dai, X.-Q.; Xu, H.-J.; Weng, J.-Q. Chin. J. Org. Chem. 2018, 38, 2807 (in Chinese); (徐雯秀, 戴小强, 徐涵 靖, 翁建全, 有机化学, 2018, 38, 2807).

[3] (a) McNally, A.; Prier, C. K.; MacMillan, D. W. C. Science 2011, 334, 1114; (b) Prier, C. K.; MacMillan, D. W. C. Chem. Sci. 2014, 5 , 4173.

[4] (a) Miyake, Y.; Nakajima, K.; Nishibayashi, Y. J. Am. Chem. Soc. 2012, 134, 3338; (b) Kohls, P.; Jadhav, K.; Pandey, G.; Reiser, O. Org. Lett., 2012, 14, 672; (c) Espelt, L. R.; Wiensch, E. R.; Yoon, T. P. J. Org. Chem. 2013, 78, 4107; (d) Lin, S.-X.; Sun, G.-J.; Kang, Q. Chem. Commun. 2017, 53, 7665.

[5] Miyake, Y.; Nakajima, K.; Nishibayashi, Y. Chem. Eur. J. 2012, 18 , 16473.

[6] (a) Uraguchi, D.; Kinoshita, N.; Kizu, T.; Ooi, T. J. Am. Chem. Soc. 2015, 137, 13768; (b) Fava, E.; Millet, A.; Nakajima, M.; Loescher, S.; Rueping, M. Angew. Chem. Int. Ed. 2016, 55, 6776.

[7] (a) Wang, C.-Y.; Qin, J.; Shen, X.-D.; Riedel, R.; Harms, K.; Meggers, E. Angew. Chem. Int. Ed. 2016, 55, 685; (b) Li, W.-P.; Duan, Y.-Q.; Zhang, M.-L.; Cheng, J.; Zhu, C.-J. Chem. Commun. 2016, $52,7596$.

[8] Zhou, Q.-Q.; Liu, D.; Xiao, W.-J.; Lu, L.-Q. Acta Chim. Sinica 2017, 75,110 (in Chinese); (周泉泉, 刘丹, 肖文精, 陆良秋, 化学学报, 2017, 75, 110).

[9] Itoh, K.; Kato, R.; Kinugawa, D.; Kamiya, H.; Kudo, R.; Hasegawa, M.; Fujii, H.; Suga, H. Org. Biomol. Chem. 2015, 13, 8919.

[10] (a) Rau, H. Chem. Rev. 1983, 83, 535; (b) Inoue, Y. Chem. Rev. 1992, 92, 741; (c) Peters, K. S. Adv. Photochem. 2002, 27, 51; (d) Fagnoni, M.; Dondi, D.; Ravelli, D.; Albini, A. Chem. Rev. 2007, 107, 2725; (e) Hoffmann, N. Chem. Rev. 2008, 108, 1052; (f) Bach, T.; Hehn, J. P. Angew. Chem., Int. Ed. 2011, 50, 1000.

[11] (a) Szostak, M.; Fazakerley, N. J.; Parmar, D.; Procter, D. J. Chem. Rev. 2014, 114, 5959; (b) Zheng, X.; Huang, P.-Q. Prog. Chem. 2018, 30, 528.

[12] Ye, C.-X.; Melcamu, Y. Y.; Li, H.-H.; Cheng, J.-T.; Zhang, T.-T.; Ruan, Y.-P.; Zheng, X.; Lu, X.; Huang, P.-Q. Nat. Commun. 2018, 9 , 410.

[13] Zhong, Y.-W.; Xu, M.-H.; Lin, G.-Q. Org. Lett. 2004, 6, 3953.

[14] For reviews on vicinal diamines, see: (a) Lucet, D.; Gall, T. L.; Mioskowski, C. Angew. Chem. Int. Ed. 1998, 37, 2580; (b) Visa, A.; Pradilla, R. F.; García, A.; Flores, A. Chem. Rev, 2005, 105, 3167; (c) Kotti, S. R. S. S.; Timmons, C.; Li, G. Chem. Biol. Drug. Des. 2006, 67, 101; (d) Cardona, F.; Goti, A. Nat. Chem. 2009, 1, 269; (e) Grygorenko, O. O.; Radchenko, D. S.; Volochnyuk, D. M.; Tolmachev, A. A.; Komarov, I. V. Chem. Rev. 2011, 111, 5506; (f) Zhu, Y.-G.; Cornwall, R. G.; Du, H.-F.; Zhao, B.-G.; Shi, Y. Acc. Chem. Res. 2014, 47, 3665. 